\title{
Valve health monitoring system utilizing smart instrumentation
}

\author{
Scott L. Jensen ${ }^{\mathrm{a}^{*}}$, George J Drouant ${ }^{\mathrm{b} \dagger}$ \\ ${ }^{a}$ NASA, Engineering and Science Directorate, EA31 Bldg 3225, Stennis Space Center, MS 39529 \\ ${ }^{b}$ NASA Technical Operations Groun/Engineering. Bldg 3226. Stennis Space Center. MS 39529
}

\begin{abstract}
The valve monitoring system is a stand alone unit with network capabilities for integration into a higher level health management system. The system is designed for aiding in failure predictions of high-geared ball valves and linearly actuated valves. It performs data tracking and archiving for identifying degraded performance. The data collection types are: cryogenic cycles, total cycles, inlet temperature, outlet temperature, body temperature, torsional strain, linear bonnet strain, preload position, total travel, and total directional changes. Events are recorded and time stamped in accordance with the IRIG B True Time. The monitoring system is designed for use in a Class 1 Division II explosive environment. The basic configuration consists of several instrumentation sensor units and a base station. The sensor units are self contained microprocessor controlled and remotcly mountable in three by threc by two inches. Each unit is potted in a fire retardant substance without any cavities and limited to low operating power for maintaining safe operation in a hydrogen environment. The units are temperature monitored to safeguard against operation outside temperature limitations. Each contains $902-928 \mathrm{MHz}$ band digital transmitters which meet Federal Communication Commissions requirements and are limited to a 35 foot transmission radius for preserving data security. The basestation controller correlates related data from the sensor units and generates data event logs on a compact flash memory module for database uploading. The entries are also broadcast over an Ethernet network. Nitrogen purged National Electrical Manufactures Association (NEMA) Class 4 Enclosures are used to house the base-station.
\end{abstract}

Keyword: Health monitoring, valve monitoring, data tracking, data logger, instrumentation, sensors, wireless, hydrogen environment

\section{INTRODUCTION}

Previous propulsion testing has incurred unexpected schedule delays and costs due to untimely maintenance, repair or replacement of facility valves. The serviceable conditions of valves utilized in the Propulsion Test Facility are critical. These significant pieces of test equipment must be closely monitored and maintained in safe working order. The existing operational data available on the facilities' test critical valves make their life expectation unpredictable. Life and failure predictions can be greatly improved through added valve instrumentation. Conventional instrumentation methods have been cost prohibitive and incapable of being mounted in the desired locations. Applying new integrated electronic technology in the form of smart sensors organized in a data tracking system is an effective way of overcoming these limitations and providing a method of implementing a valve health monitoring system.

It is cost prohibitive to obtain spares for existing valves that have replacement periods of over one year and repair times of approximately six months. It is becoming a necessity to track the life of linearly-actuated valves and high-geared ball valves to efficiently perform testing. Employing a valve monitoring system would aid in managing valve maintenance and failures.

\section{METHODOLOGY}

A knowledge base on valve operational characteristics would provide insight into life duration and failure predictions. The data tracking and archiving which would be most beneficial in building a knowledge base were identified as:

*Scott.L.Jensen@nasa.gov; NASA Engineering and Science Directorate, Mail Stop EA31

George.Drouant@ssc.nasa.gov; NASA Technical Operations Group/Engineering 
cryogenic valve cycles, inlet temperature, outlet temperature, valve body temperature, total valve cycles, cryogenic valve cycles, torsional strain, linear bonnet strain, valve preload position. Monitoring systems on the market were unable to meet all the necessary attributes for implementation in our facilities propulsion test environment. A valve monitoring system was designed to record events associated with the previously mentioned instrumentation collection tvpes. The IRIG B True Time convention was adopted as the required data synchronization protocol since it is used throughout many propulsion test facilities. Therefore, the data events are time stamped in accordance with the facility IRIG B True Time to allow data synchronization within 1 millisecond. The data is then organized into a text file which can be uploaded into the pre-existing tacility database.

\section{TECHNICAL DESCRIPTION}

\subsection{The monitoring system fundamental configuration}

The valve monitoring system's fundamental configuration consists of a single base station and multiple sensor units. Each sensor unit is assembled for a particular monitoring function. The units were designed for torsional strain, linear strain, temperature, valves cycles and valve position monitoring. These were identified to provide data on the necessary valve attributes that would indicate valve failures and degraded performance. All the sensor units are functionally encapsulated and the detected events are coordinated through the base station. The base station is organized into the main base station controller, flash memory interface, external communication module, bus interface, and several receiver modules. Each receiver unit has a unique address and it is matched up with a remote sensor unit's transmitter. The sensor units are organized into a sensor controller, power regulator, two battery packs, transmitter module, wake-up module, and several instrumentation modules. The configuration is shown in Figure 1.
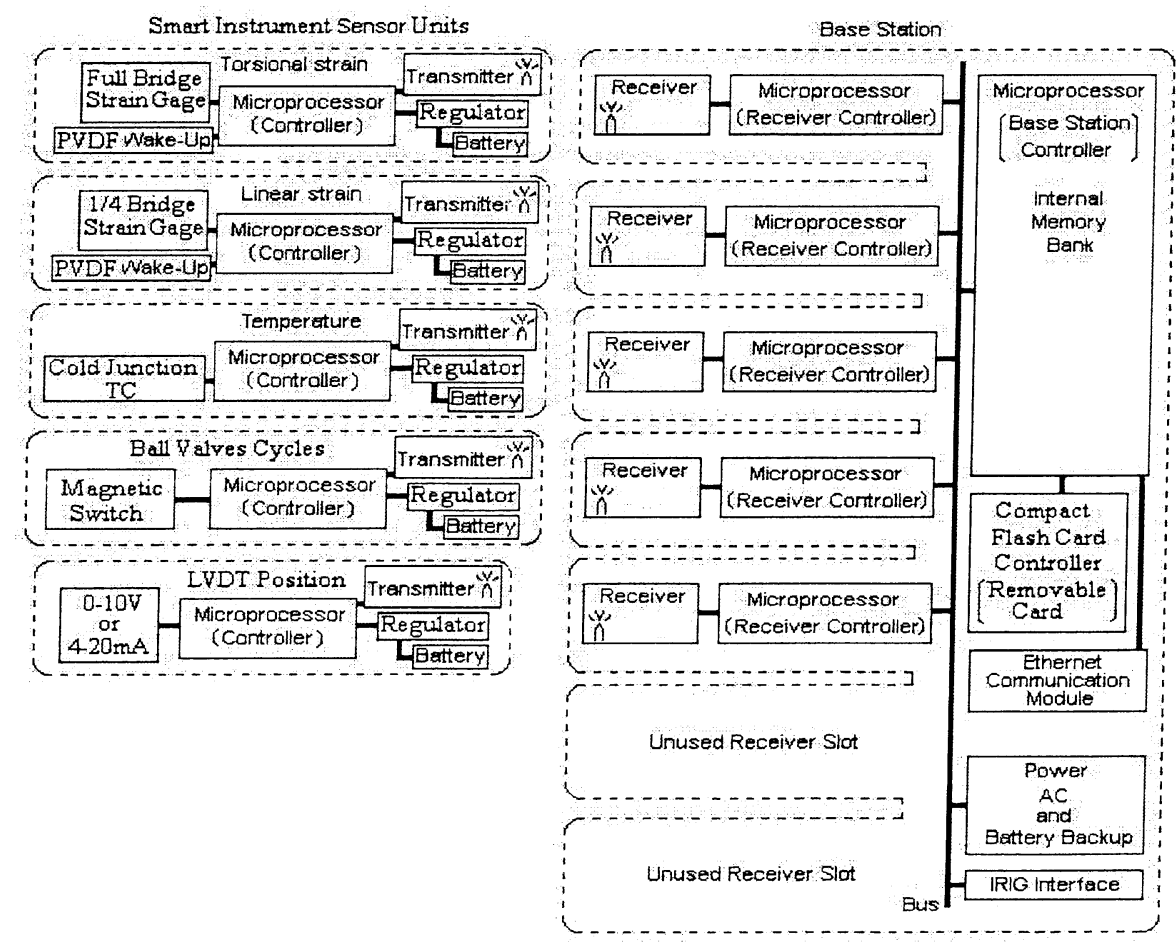

Figure 1: Sensor units' organizational configuration.

The high-geared ball valves and the linearly actuated valves were targeted for health monitoring with this system. The system may be configured for other monitoring duties and not limited to these valve types. The placement and utilization of the sensors are critical for the system's monitoring capabilities. 
A method for determining how much a ball valve has been utilized is accomplished by tracking the total number of valve cycles. The number of valve cycles correlated with valve failures provides a basis for making valve life predictions. Another consideration in predicted valve life duration is the number of cycles under cryogenic service. The service duration for valves at cryogenic temperatures will be different from ambient operation. The determination of cryogenic or ambient operation will be made hy correlating the inlet and outlet temperatures with each vaive cycic. The monitoring system was constructed with a torsional strain sensor unit. The unit was made small enough to be mounted on the ball valve shaft. The clearance necessary for this mounting location is approximately 2 by 3 by 3 inches. The motion of the shaft forces the sensor unit to use a wireless interface. The monitoring system is designed tor use in a Class 1 Division II hydrogen environment per National Electrical Code Article 501 ${ }^{1}$. All the units are potted in a hydrogen compatible material. The potting material used is blue-epoxy flame retardant 832FRB from M.G.

Chemicals ${ }^{2}$. A sensor unit was designed for counting the cycles the ball valve has undergone while in service. The switch sensor unit uses five magnetic position switches for indicating the ball valve state. A Temperature sensor unit was constructed to monitor the inlet and outlet temperatures utilizing cold junction referenced thermocouples (TC). A total of four sensor units are adequate for monitoring a single high-geared ball valve. The typical sensor unit mounting locations on high-geared ball valves are shown in Figure 2.

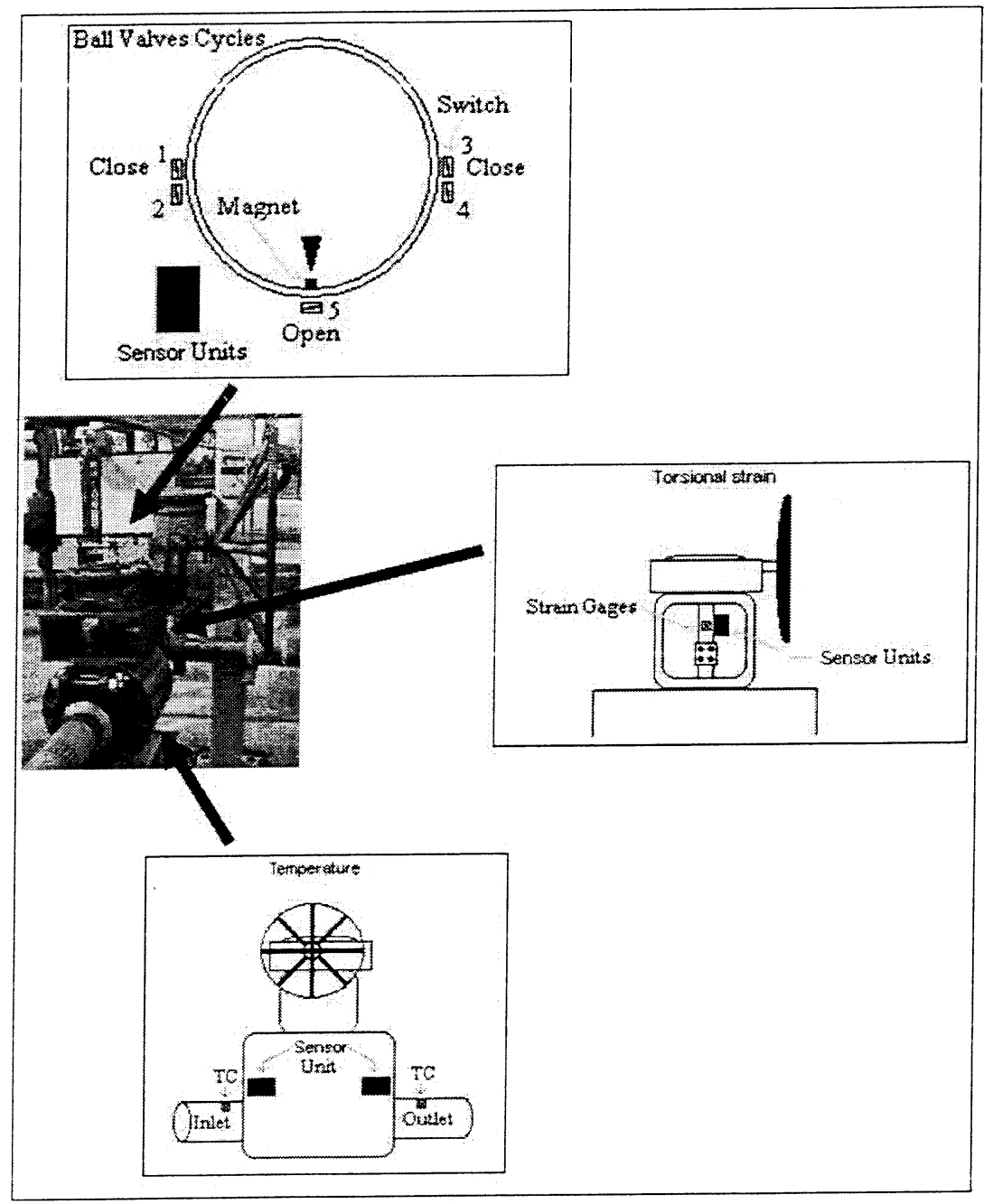

Figure 2: Typical placement of sensor units on high-geared ball valves.

The monitoring system was also designed to observe linearly-actuated valves. The valve plug seating was targeted as a significant point of failure along with packing and seal breakdown. Irregularities in the force during valve cycling can 
be determined by logging the strains on each side of the valve bonnet. A linear strain sensor unit was developed for monitoring the axial strain for that application. Observing irregularities in this force will indicate issues involving the linear valve stroke, which will help denote signs of failure in the valve packing or seals. Another sensor unit was designed for tracking the total distance of valve travel and number of directional changes over its duration of service. A signal sensor unit was designed to unohtrusively interface with the Linear Variable Displacement Transducer (LVDT) signal from the internal servo controller. The LVDT signal provides a highly accurate valve position within $0.01 \%$ of the valve stroke. The inlet and outlet temperatures are monitored with a temperature sensor unit identical to the one used to monitor temperatures on the high geared ball valve. The temperature sensor unit can also be used to monitor the valve body temperatures. The typical sensor unit mounting locations on linearly-actuated vaives are shown in Figure 3.

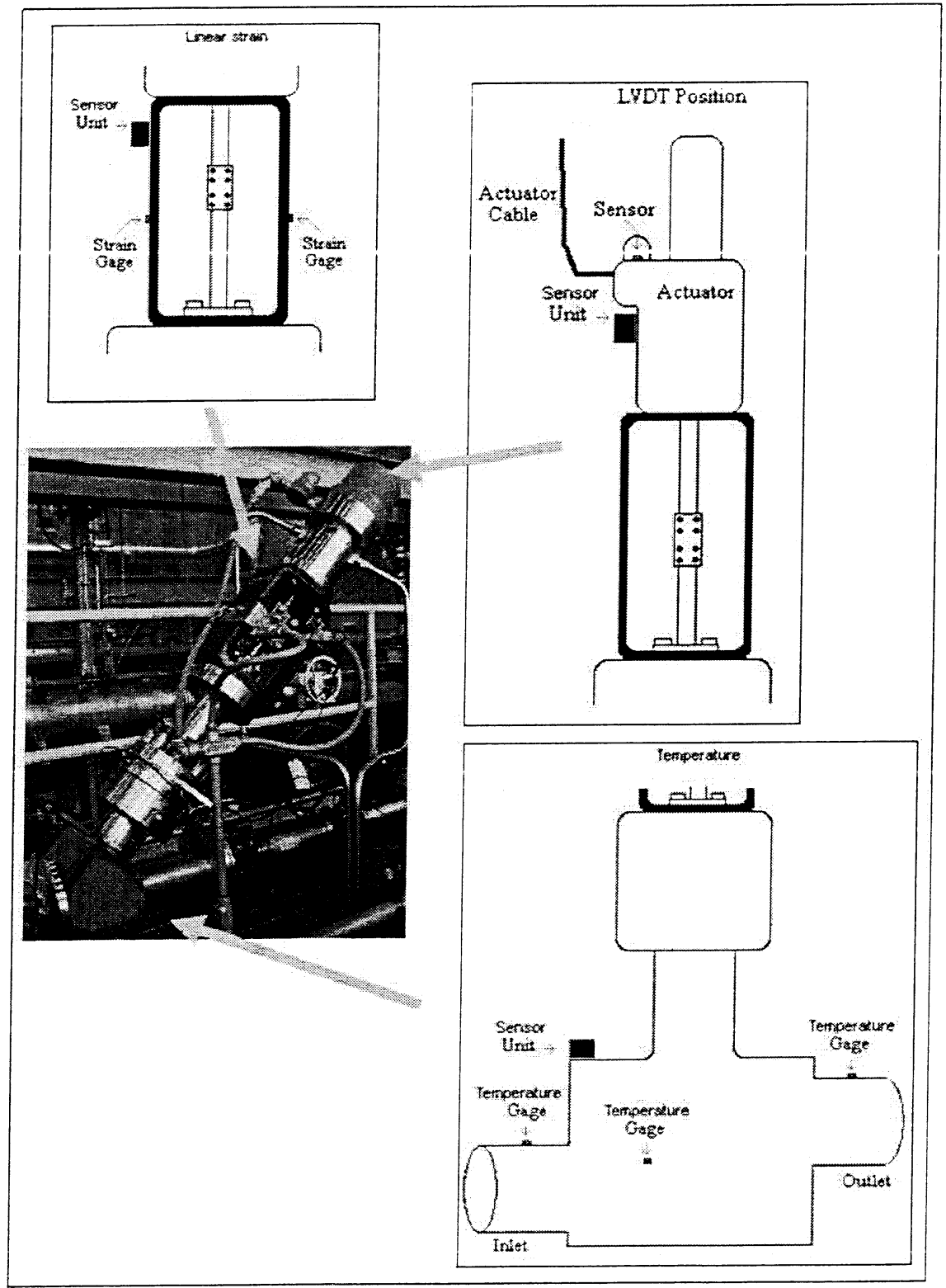

Figure 3: Typical placement of sensor units on linearly-actuated ball valves.

\subsection{The monitoring system base}


The foundations of the units are microprocessor controller circuits which utilizes the PIC16F887A microprocessor ${ }^{3}$ from Microchip Technology Incorporated. This circuit was designed for a dual use function as either the receiver module within the base station or the sensor unit microprocessor controller. The function is determined by the way the integrated circuit board is populated. It contains an oscillator for a 4 megahertz processor speed and another 32.768 kilohertz oscillator for a precision clock. It has a human interface switch and a light emitting diode indicator for performing setup processes and checking battery life. An embedded magnetic reed switch is utilized to eliminate any arcing from switch contact closure. It also has a reboot switch when used as a receiver module.

The TMP36GS temperature component is positioned near the microprocessor for safeguard against operation outside the processor's temperature limitations. It reads the operational temperature within one degree Celsius. It is also positioned directly beneath the battery packs to avoid heating the battery packs beyond the hydrogen explosive limits. The battery packs are not utilized in the receiver module configuration, but the operational temperatures are still monitored for the processor. This internal temperature monitoring component is put into shutdown mode when the sensor unit is in sleep mode to help conserve battery life.

The Linx HP3 transmitter model TXM-900-HP3-PPS ${ }^{4}$ is employed in this circuit when configured as the sensor unit. The Linx HP3 receiver model REX-900-HP3-PPS ${ }^{4}$ is employed when configured as the receiver module. These provide low-power 902-928 MHz band digital transmissions which meet Federal Communication Commissions requirements. The transmission power is set to iimit transmissions to a 35 foot radius for preserving data security. Each receiver/transmitter pair is set to a unique address. The addressing scheme implemented provides eight unique addresses. This restricts each monitoring system to eight sensor units. This is ample for monitoring a single valve, but provisions for enabling over two hundred unique addresses are in place for future implementation.

The microprocessor controller circuit also contains several connections for interfacing to the other sensor unit circuitry. In addition to this, an onboard programming and serial communication interface port header and a communication bus interface header are enclosed within this circuit. The base station's Bus allows a varying number of receiver units to be connected. Each receiver unit has a unique address and it is matched up with a remote sensor unit.

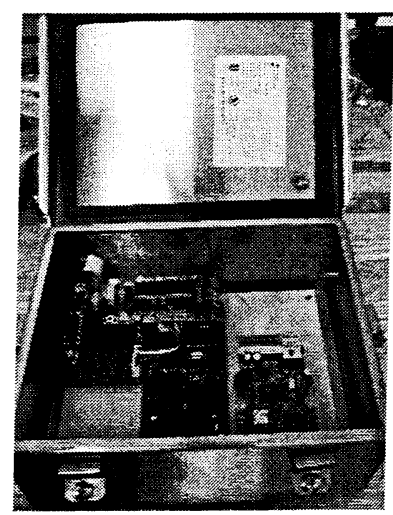

Figure 4: The base and enclosure

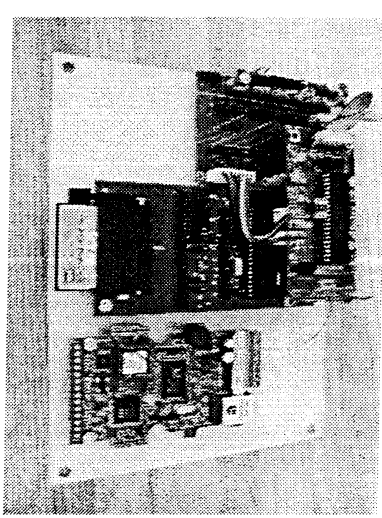

Figure 5: The base station

\subsection{The monitoring system sensor units}

The microprocessor, regulator, transmitter and internal temperature circuitry are common to all the sensor units. The piezoelectronic wake-up circuitry is only necessary in the strain sensor units. The sensor units are constructed in a manner to minimize modifications for implementing different instrumentation types. However, each instrumentation sensor type requires a unique instrumentation circuit. This is accomplished by using a uniform interface for instrumentation circuit boards of all desired types. The only modifications required in the other sensor circuitry are setting the transmitter address, routing of the secondary regulator, and insertion of the wake-up circuit. This allows for developing new instrumentation monitoring types by devcloping a new instrumentation circuit board and eliminating redevelopment of other components. 
The sensor unit's wake-up circuitry uses a laminated piezoelectronic sheet for highly sensitive vibration detection. The sensitivity is adjustable prior to potting the sensor unit. LDT series vibration detector by The Measurement Specialties Inc. ${ }^{5}$ is used to detect any valve movements. The piezoelectronic laminate produces a voltage capable of waking the microprocessor from sleep mode without any power required from the sensor unit's batteries. This allows the sensor unit to remain in sleep mode with little power consumption but continuously monitor a valve for any events which would require its other instrumentation. This is a key element in extending the sensor unit's battery life.

I he sensor unit's power regulator circuitry takes advantage of the teatures provided by the dual input smart battery backup regulator from Linear Technology ${ }^{6}$. Two of the devices are use to deliver power from four batteries. Both regulators can supply power to the entire sensor unit for extended battery operation while maintaining a seamless transfer of supplied power from each battery. When necessary, one can be routed to instrumentation providing excellent isolation for precision instrumentation and noise reduction. The regulator has a power down feature to reduce the power consumption when it is not needed. The regulator also is capable of monitoring the state of the batteries. This allows the microprocessor to appropriately manage power utilization. The battery levels are tested during the update phase of the microprocessor. A data event is transmitted to the base stations indicating required maintenance if a low state is encountered.

The temperature sensor unit utilizes a cold junction referenced thermocouple instrumentation circuit. The circuit uses a monoii hic inermocoupie ampiifier chip with coid junction compensation presrimmed for Type $\mathrm{K}$ inermocoupies frum Analog Devices ${ }^{7}$. The temperature sensor unit accepts two Type $\mathrm{K}$ thermocouples. The microprocessor reads in an analog signal from the thermocouple and converts it to degrees Fahrenheit. A table lookup algorithm is implemented to obtain an accuracy of plus or minus one degree Fahrenheit. The Omega Engineering Inc. ${ }^{8}$ Type K thermocouple conversion tables are utilized in the table lookup algorithm. The unit is capable of reading temperatures from -454 Fahrenheit to 315 Fahrenheit. The unit can also be point calibrated for a specific thermocouple through the onboard programmer interface header. The unit is pre-programmed to read a temperature every sixteen seconds, and can be adjusted as desired. Once the conversion is completed a data event containing the temperature and time stamp is transmitted to the base station.

The strain instrumentation circuit is used to monitor axial, biaxial and torsional strain types. Each strain instrumentation sensor unit can read two strain types. The placement of precision resistors in the circuit denotes the circuit as a full, half, or quarter bridge foil strain gage configuration. The quarter bridge configuration monitors the single axial bonnet strain for the linearly actuated valve. The Vishay CEA-09-125UN-350 foil strain gage ${ }^{9}$ is used in a quarter bridge configuration. The half bridge configuration monitors the same single axial bonnet strain but offers temperature compensation. The Vishay SK-09-060WT-350 foil strain gage is used in a half bridge configuration. This configuration is superior when only a single axial strain can be produced, but if any biaxial strains are expected the quarter bridge configuration must be used. A 4 micro strain resolution is obtainable from the quarter and half bridge configurations. The full bridge configuration monitors the torsional strain in the high geared ball valve. The Vishay CEA-09-250US-350 foil strain gage ${ }^{9}$ is used in a full bridge configuration. A four micro strain resolution is obtainable from the full bridge configurations. The typical ball valve requiring health monitoring uses a 2 inch solid 304 stainless rod. A $50 \mathrm{ft} / \mathrm{lb}$ torsional force on such a steel rod is accurately detectable ${ }^{3}$.

A simple magnetic reed limit switch instrumentation circuit is use for tracking the number of valve cycles performed on a high geared ball valve. The limit switch instrumentation circuit can monitor six switches with the ability to wake the sensor unit up on two switches. The determination of valve cycles performed under cryogenic temperatures is accomplished by the base station correlating a valve cycle event with the inlet and outlet temperature. The base station keeps up with totals and generates the proper data log entry.

The signal circuit was designed to unobtrusively interface with the Linear Variable Differential Transformer (LVDT) signal from the valve's internal servo controller. It enables the sensor unit to read a 4 - 20 milliamp signal or a 0 - 10 volt DC signal for reading the valve position signal. A highly sensitive magnetic sensor reads the milliamp signal by monitoring the magnetic fields produced from the current loop. The sensors used are Giant Magnetoresistance AA series analog sensors from Nonvolatile Electronics Incorporated ${ }^{10}$. The voltage signal indicates the level by use of a voltage follower circuit. Utilizing these methods ensures failures in the monitoring unit will not affect the operation of the valve. The LVDT signal provides a highly accurate valve position within $0.01 \%$ of the valve stroke. Monitoring the 
position changes denotes the total distance of valve travel and the number of directional changes. The monitoring system logs the accumulated distance traveled and total number of directional changes after each position's resultant travel. The fully closed valve position applies a predefined amount of load against the valve seat. This is referred to as the valve preload. As the valve seat incurs wear, the valve's closed position slightly changes reducing the preload. The valve position is monitored for any change in the closed position and generates a data event when necessary.

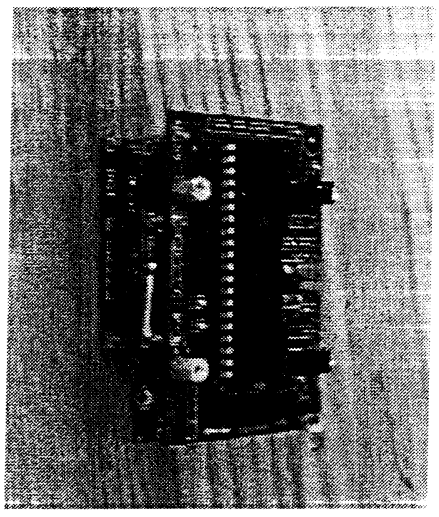

Figure 4: The un-potted sensor unit

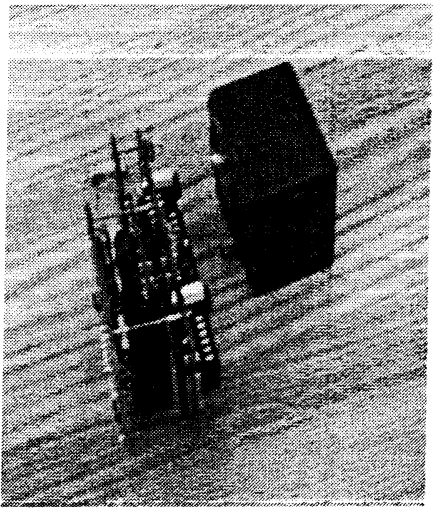

Figure 5: The sensor unit and a potted battery pack

\subsection{Additional attributes}

The valve monitoring system must be able to meet other critical criteria to be applicable to field operation. The sensor units must be very power conservative to make them maintainable. The battery packs must last for several weeks, unlike data logging systems on the market, which typically last a few days. The sensor units are believed to last four to five weeks. The largest power consuming portion is the wireless transmission device. It must be powered down for the majority of operational time. This means a receiver on the sensor unit is not an option. A one way communication protocol must be implemented. This makes time synchronization difficult to achieve. The sensor unit and base station receiver unit both have accurate oscillators which can keep time within a quarter of a millisecond for over five weeks. The sensor unit transmits its time once a day to the base station. It only needs to perform one transmission in order to maintain accurate time synchronization. The base station keeps the previous day's time synchronization if a transmission error occurs or no transmission is made. If such an event occurs an entry into the data log is made in order to identify any data corruption due to time stamping. The monitoring system has 35 days to perform a good data synchronization link-up before any time accuracy is at risk. The base station has an IRIG-B timing module which provides a digital signal to all the base station receiver units. These units synchronize the sensor unit's time to the IRIG time. When a data event occurs the sensor unit transmits the event and its time according to an internal clock. Redundant transmissions are performed to avoid data transmission errors. The base station receiver unit converts the sensor time to IRIG time. The data event is then passed to the base station's main microprocessor which correlates any other applicable data such as inlet temperature. The refined event $\log$ is then stored in a text string on a removable flash memory card and broadcasted on an Ethernet network. The flash memory access units are made with a Compact Flash Development Kit from ARMA Design Inc ${ }^{11}$. The microprocessor aboard the Compact Flash board is use as the base station's main controller. The data broadcasting performed by an off-the-shelf Ethernet embedded controller unit called an I-7188E Module which is manufactured by ICS DataCom ${ }^{12}$. The implementation of other network communication transmissions can be accomplished by using the desired communication module ${ }^{6}$.

\section{CONCLUSIONS}

The valve monitoring system is a feasible solution for helping maintain high-geared ball valves and linearly actuated valves within the Propulsion Test Facilities. It helps avoid untimely failures by flagging degraded performance. However, this approach is not feasible for determining degraded operation during a propulsion tcst duc to the highly dynamic conditions. It may take several seconds to correlate the data with an event and transmit an alert through the system. However, a considerable advantage can be obtained by monitoring valve performance over the operational life 
of the valve. Post event detection can be accurately related to other events and an operational history developed. A higher level facility monitoring system would be able to automatically utilize the operational histories.

\section{REFERENCES}

1. National Fire Protection Association National Electrical Code International Electrical Code Series, Quincy, Massachusetts USA 02169-7471.

2. MG Chemicals Inc., www.mgchemicals.com, 193rd Street, Surrey, BC, Canada V4N 4E7.

3. Microchip Technology Inc. "PIC16F87XA Data Sheet 28/40/44-Pin Enhanced Flash Microcontrollers", www.microchip.com, West Chandler Boulevard, Chandler, Arizona, USA 85224-6199.

4. Linx Technologies Inc., www.linxtechnologies.com, South East Ashley Place, Grants Pass, OR 97526.

5. Measurement Specialties Inc., www. meas-spec.com, Lucas Way, Hampton, VA 23666.

6. Linear Technology Corporation, www.linear.com, McCarthy Boulevard, Milpitas, CA 95035-7417.

7. Analog Devices Corporation, www.analog.com, P. O. Box 9106, One Technology Way, Norwood, MA, 02062-9106.

8. OMEGA Engineering Inc., www.omega.com, P. O. Box 4047, One Omega Drive, Stamford, Connecticut 06907-0047.

9. Vishay Measurements Group Inc., P.O. Box 27777, Raleigh, NC 27611.

10. NVE Corporation, www.nve.com, Valley View Road, Eden Prairie, MN 55344-3617.

11. ARMA Design "Compact Flash Card Development Kit Manual”, Ferris Square, Suite E, Sand Diego, CA 92121.

1) ICS DataCom "T-7188F. Hardware Manual" Commerce Circle, Pleasanton, California 94588

The use of trademarks or names of manufacturers in the report is for accurate reporting and does not constitute an official endorsement, either expressed or implied, of such products or manufacturers by the National Aeronautics and Space Administration. 


\title{
Valve Health Monitoring System Utilizing Smart Instrumentation
}

\author{
By: \\ Scott L Jensen \\ AST, Electronics Instrumentation Systems
}

NASA, Stennis Space Center

Engineering and Science Directorate, Design and Analysis Division 


\section{Valve Health Monitoring System Utilizing Smart Instrumentation}

- Previous testing has incurred unexpected $>$ Schedule delays

$>$ Maintenance costs

- Due to valve $>$ Repair

$>$ Replacement
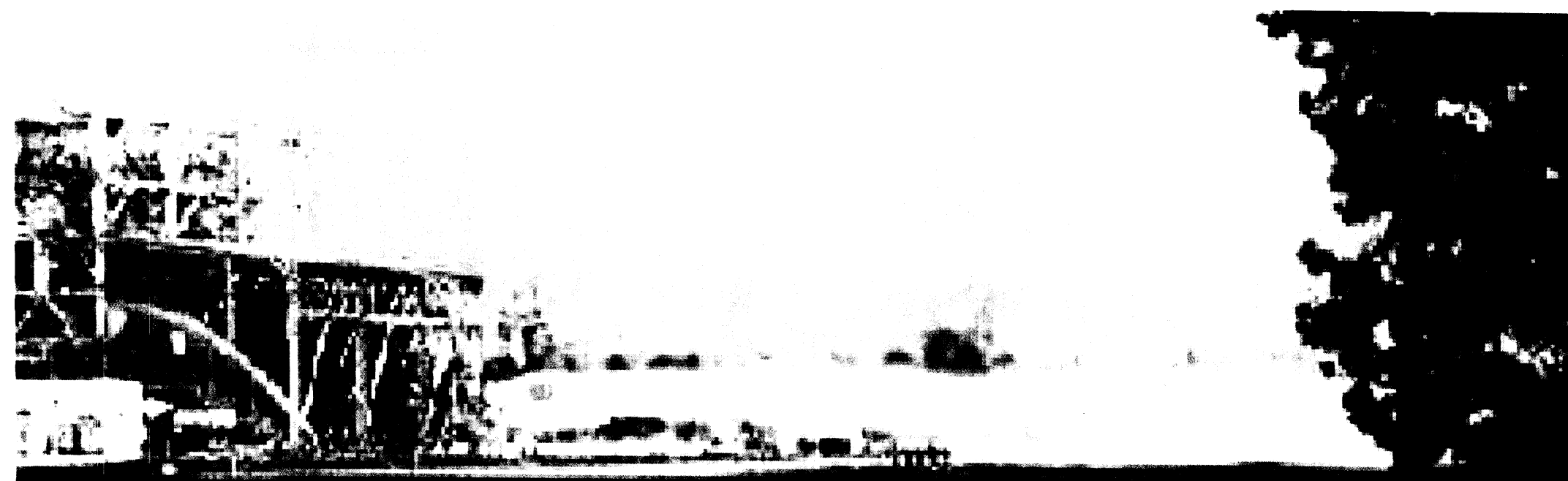

TRW 650K E1 Test Stand July 2000 


\section{Valve Health Monitoring System Utilizing Smart Instrumentation}

Need to track hardware life requires implementation of monitoring systems

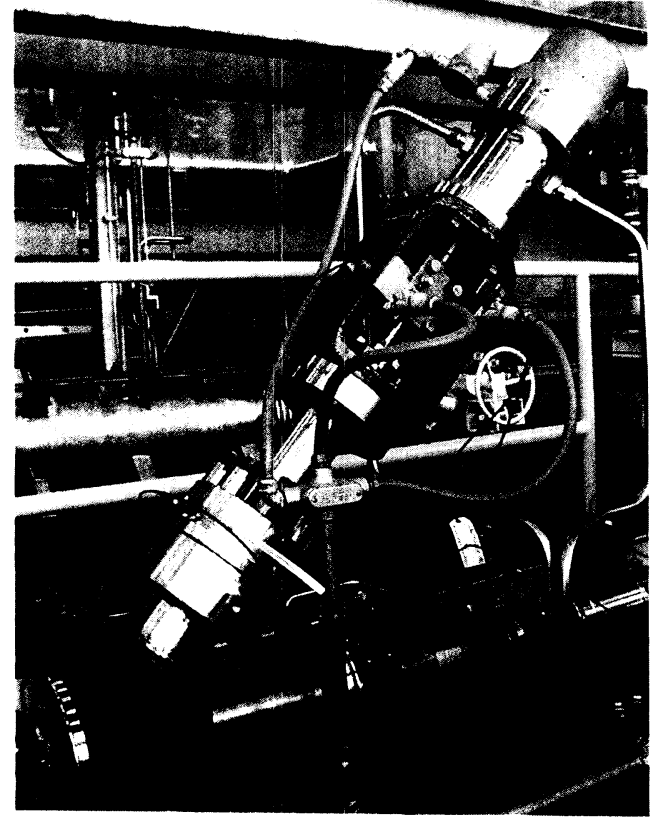

Linearly Actuated Valve
Valve monitoring for:

$>$ High-Geared Ball Valves

$>$ Linearly Actuated Valves

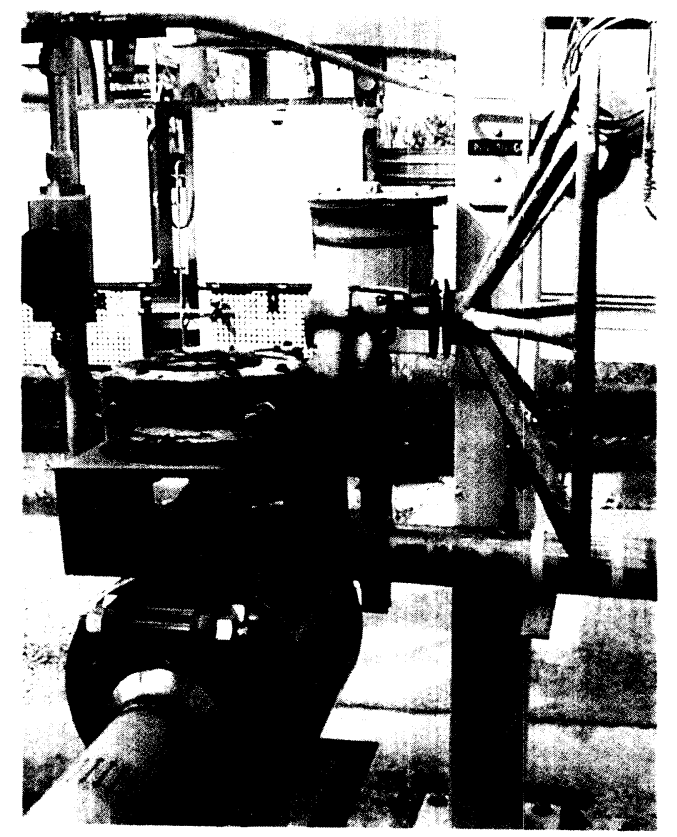

High-Geared Ball Valve

Scott Jensen 3 $03 / 10 / 2006$ 


\section{Beneficial Data Tracking Types}

- High-Geared Ball Valves

$>$ Torsional shaft strain

$>$ Total valve cycles

$>$ Cryogenic valve cycles

$>$ Inlet temperature

$>$ Outlet temperature

$>$ Body temperature
- Linearly Actuated Valves

$>$ Linear bonnet strain

$>$ Total linear travel

$>$ Total directional changes

$>$ Valve preload position

$>$ Inlet temperature

$>$ Outlet temperature

$>$ Body temperature 


\section{Sensor Placement for High-Geared Ball Valves}
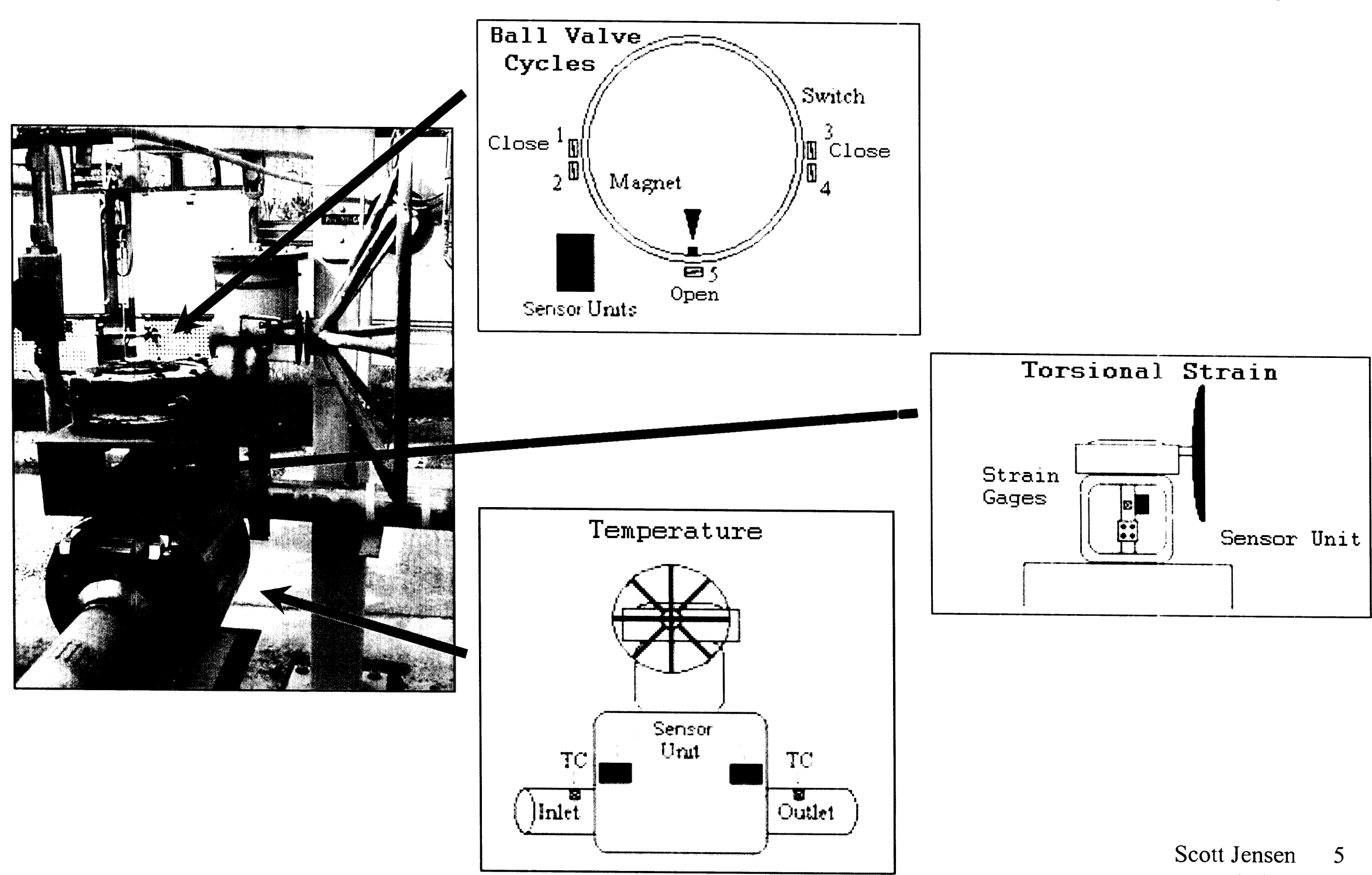


\section{Sensor Placement for Linearly Actuated Valves}
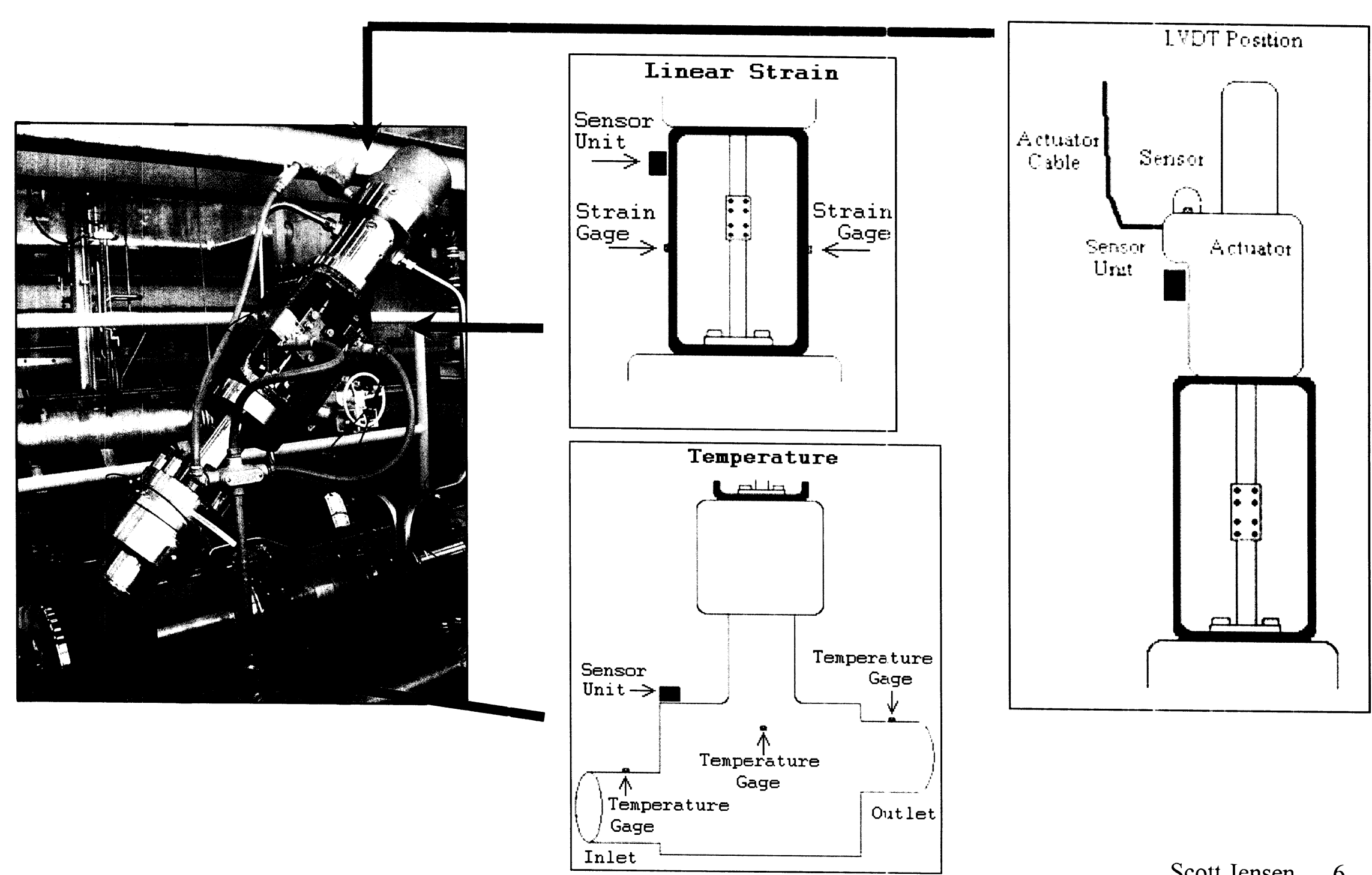

03/10/2006 


\section{Valve Monitoring System Fundamental Configuration}

Sensor Units

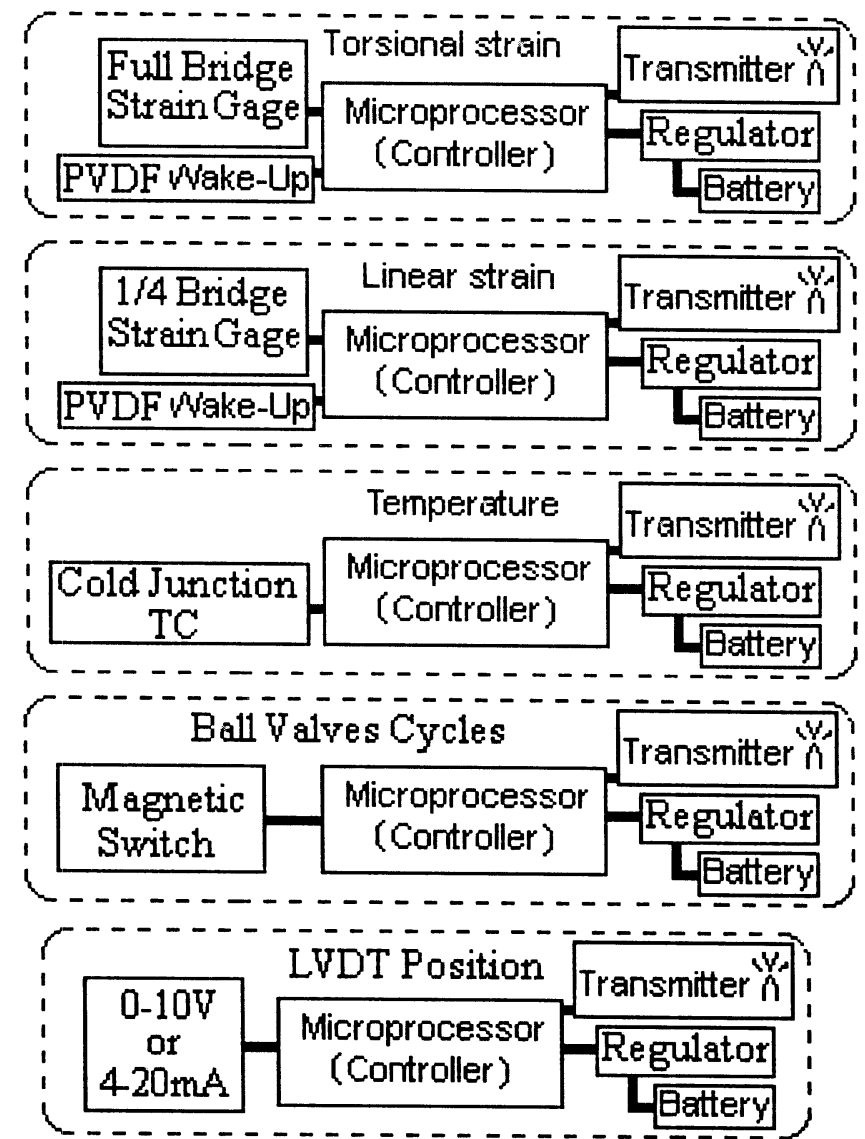

Base Station

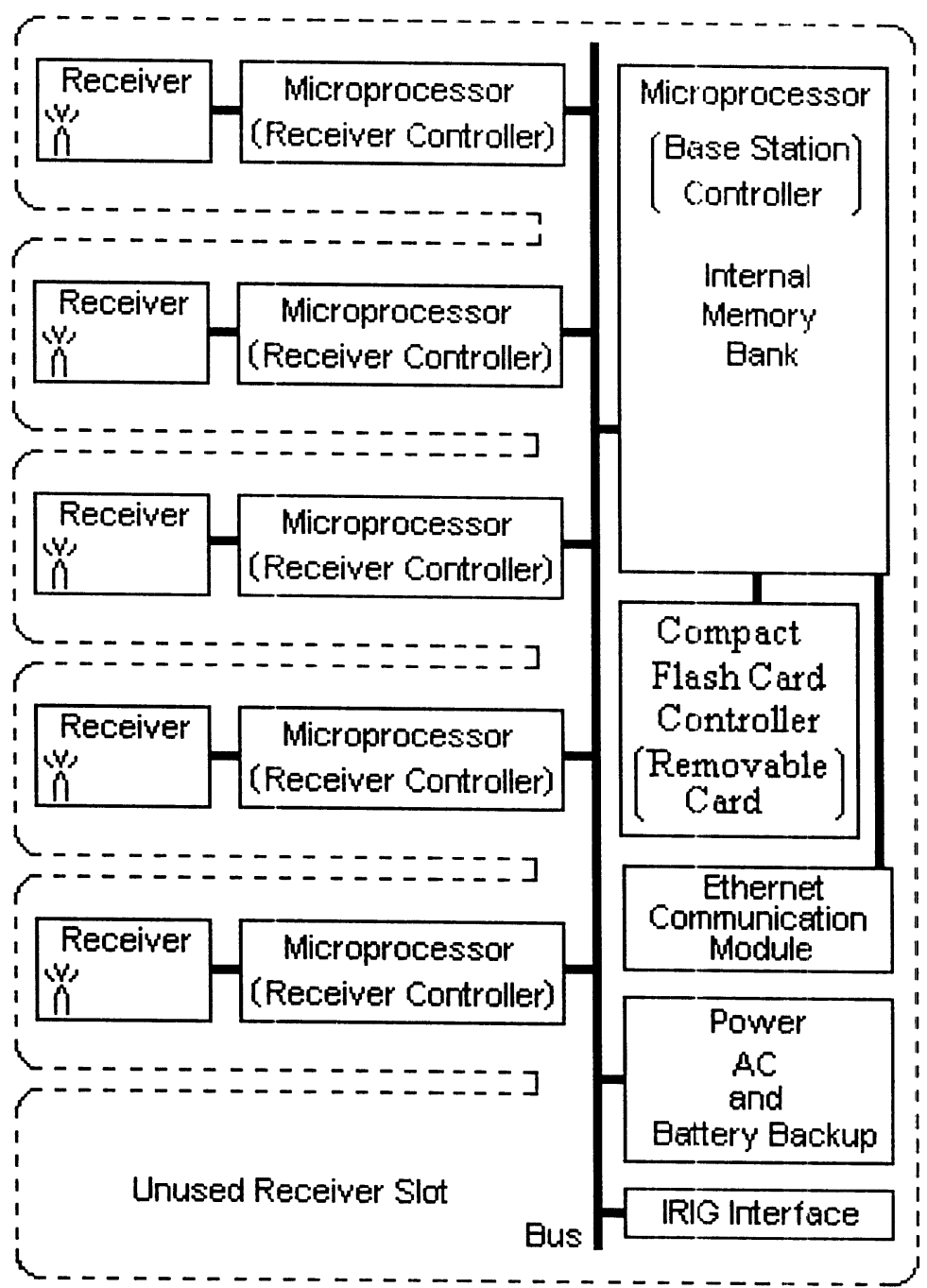

Scott Jensen 7

03/10/2006 


\section{Valve Health Monitoring System Essential Capabilities}

- Operate in Confined Locations

- Safe Operation in Explosive Environment

- Extremely Low Power Consumption

- Data Correlation

- Accurate Data Synchronization

- Automatic and Manual Data Access

- Simple Setup and Maintenance

- Expandable System

- Cost Effective Manufacturability

- Adaptive for Future Monitoring Tasks 


\section{Valve Health Monitoring System Essential Capabilities}

- Confined Locations

- Sensor Size

$2 \frac{1}{2} \times 3 \times 4$ inch

- Wireless

35 foot transmission radius

Added data security

902-928 $\mathrm{MHz}$ band

Compliance with Federal

Communication Commission

- Battery Powered

Two battery packs

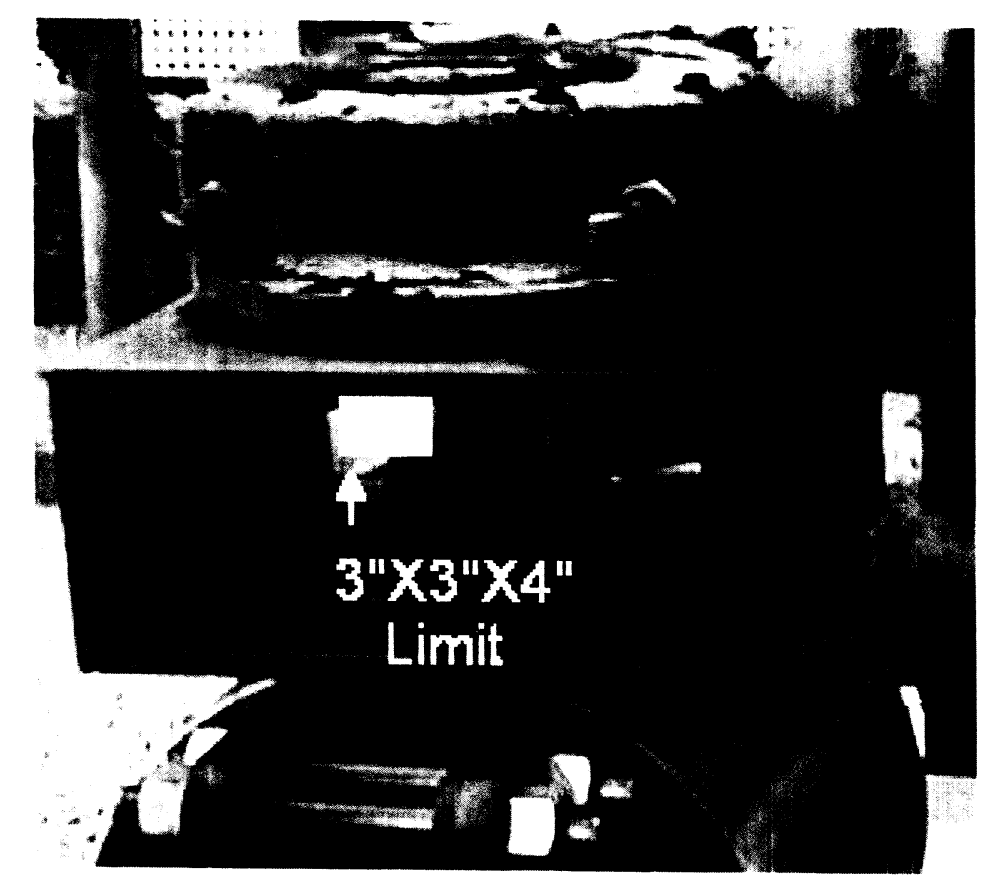

with two 9 Volts supplies 


\section{Valve Health Monitoring System Essential Capabilities}

- NEC Class I Division II Group B Hazardous Environment

- Compliance with NEC article 501

- Enclosed in Potting

Blue-epoxy flame retardant 832FRB M.G. Chemicals

- Internal temperature monitoring Shutdown at $150^{\circ} \mathrm{F}$

- No exposed arcing points

- Limited operational power

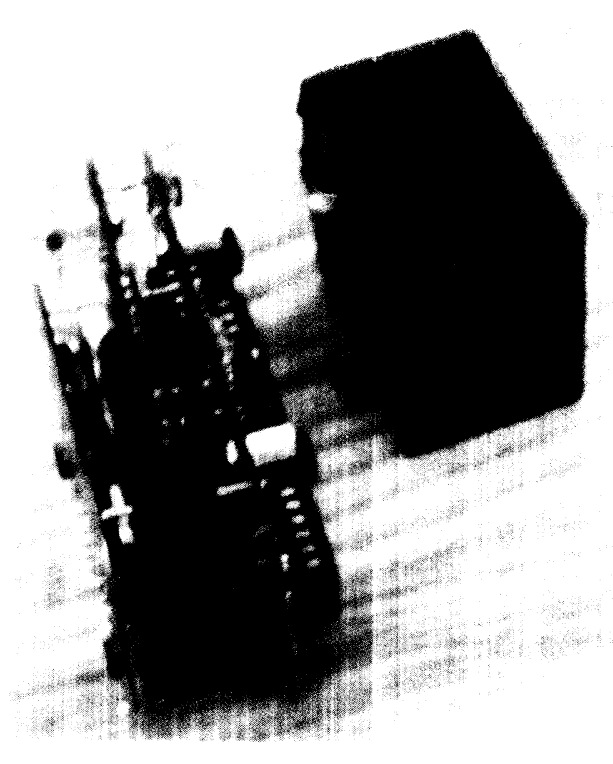

9 Volts at 250 milliamp

- No exposed cavities 


\section{Valve Health Monitoring System Essential Capabilities}

- Extremely Low Power Consumption

- One-Way Communications

Linx HP3 transmitter and receiver modules

- Microprocessor Sleep Mode

- Piezoelectronic Wake-Up Circuitry

Measurement Specialties LDT series

Piezoelectronic Sheet

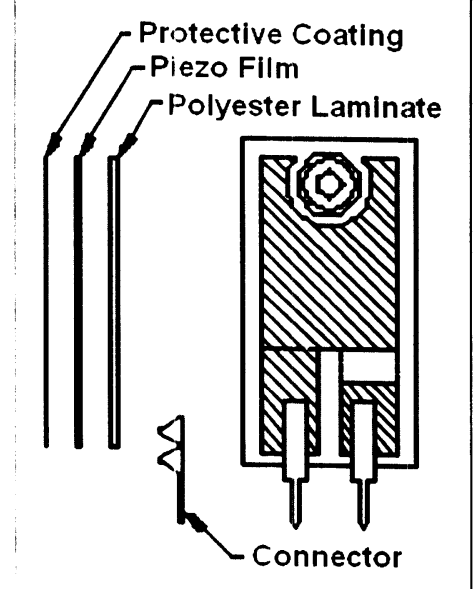

- Accurate Data Synchronization

- IRIG-B Timing Module

Facility correlation

- Communication Bus

Internal data correlation

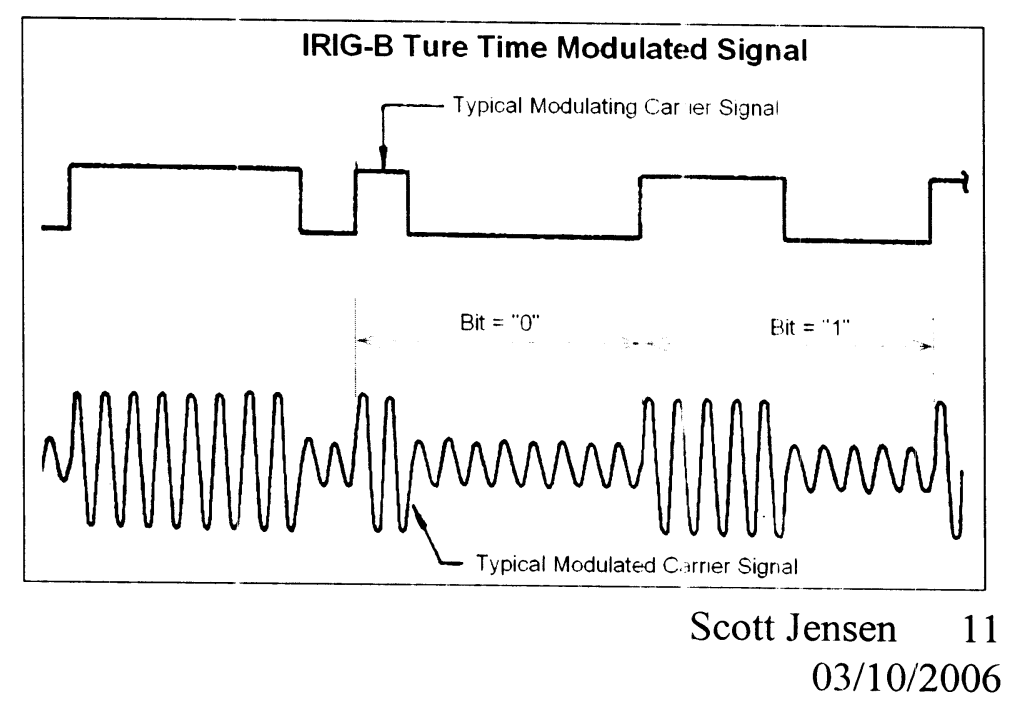




\section{Valve Health Monitoring System Essential Capabilities}

- Automatic and Manual Data Access

- Memory Storage Network Capable Compact flash card memory access ARMA Design Inc.

- Network Capable Ethernet broadcast I-7188E ICS DataCom Inc.

- Setup and Maintenance

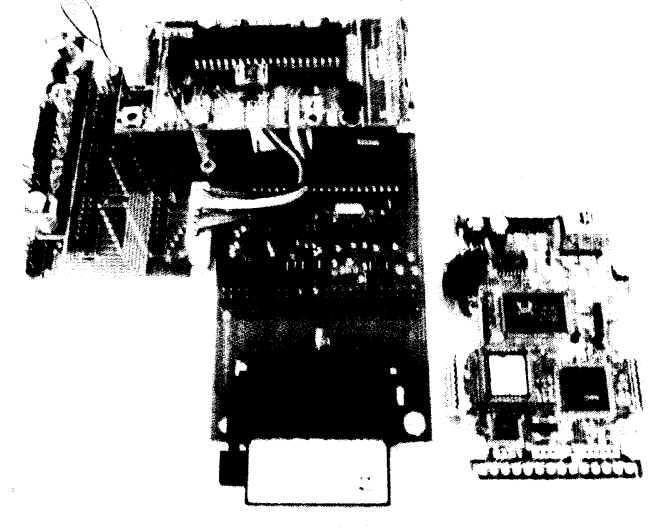

- Simple Human Interface Switch and Indicator light

- On-board Programmer Interface Serial communications Software updates

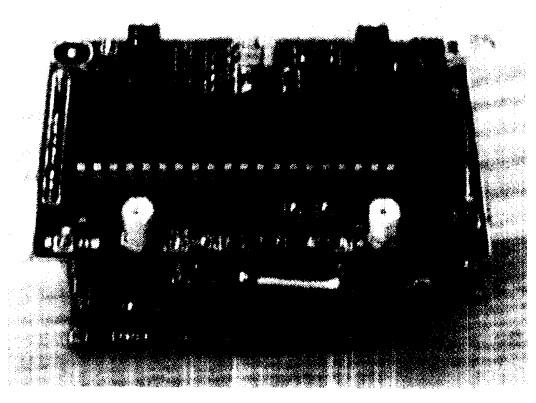




\section{Valve Health Monitoring System Essential Capabilities}

- Adaptive for Further Monitoring Tasks

- Software Upgrades

- Interchangeable Instrumentation Circuit

Each instrumentation type confined to a single circuit board Normal interface allows for further instrumentation types Eliminates redeveloping common components

- Cost Effective Manufacturability

- Manufacturing Friendly

Common circuit board etching

One-sided component population

- Commonality between circuits

Reuse of board for multiple functions

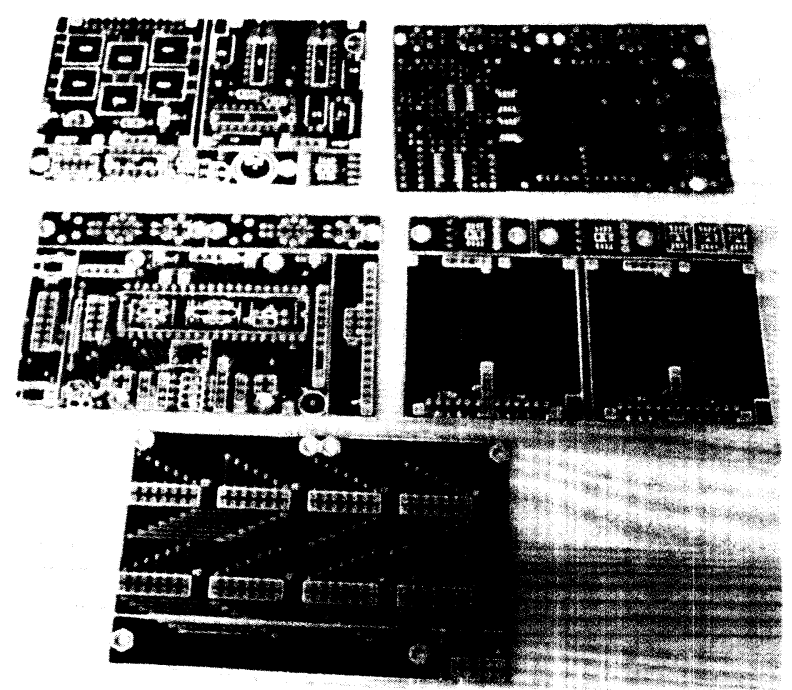




\section{Valve Health Monitoring System Common Structure}

- The Microchip Technology PIC16F877A Microprocessor is utilized in:

The Base Station controller

Sensor Units

Receiver Modules

- The main microprocessor board is identical in: The Receiver Board and Main Sensor Board

- Each receiver module utilizes the identical: The PIC16F877A microprocessor TMP36GS temperature circuitry The Linx HP3 Receiver modules

- Each sensor unit utilizes the identical: The PIC16F877A microprocessor TMP36GS temperature circuitry The Linx HP3 transmitter model Linear Technology dual battery smart regulator Dual battery packs

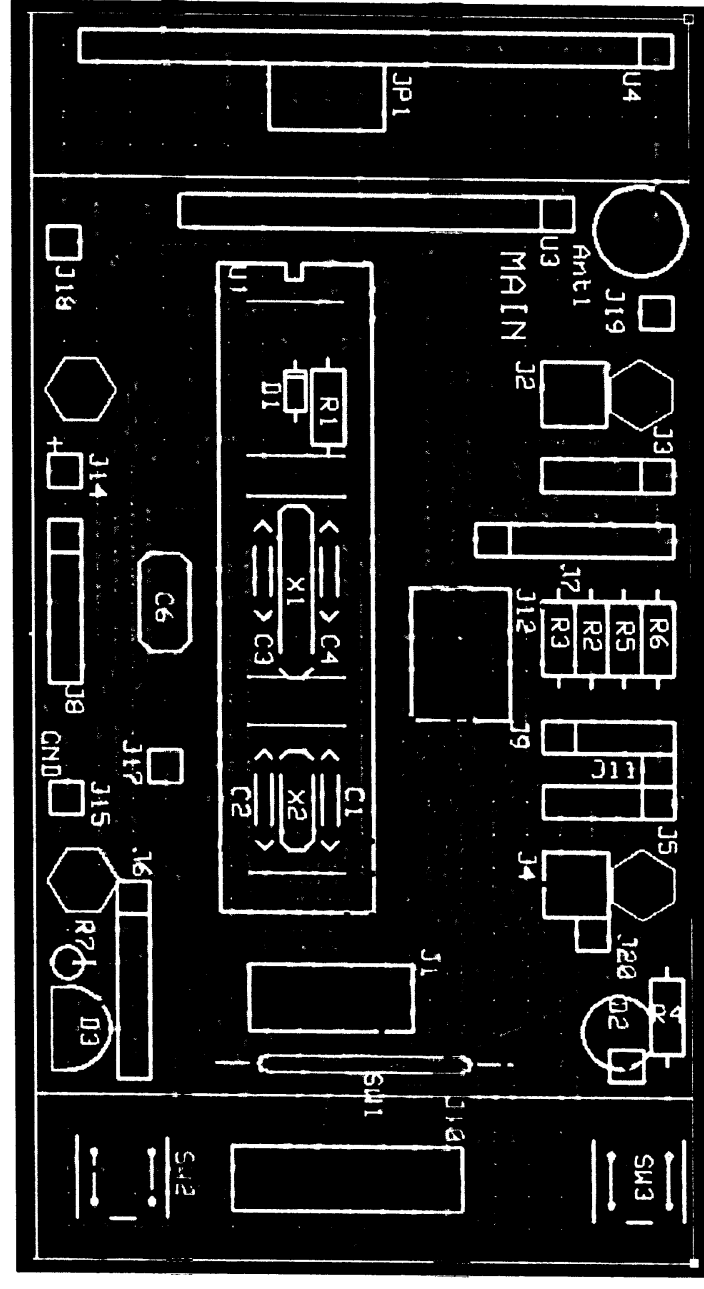

Scott Jensen 14 $03 / 10 / 2006$ 


\section{Valve Health Monitoring System Instrumentation Types}

Uniaxial Pattern

- The Type K Thermocouples Sensor (for inlet, outlet, and body temperature monitoring)

- Monolithic thermocouple amplifier from Analog Devices

- Uses cold junction compensation

- The Strain Instrumentation Sensor (for bonnet and torsional strain monitoring)

- Axial Strain by a Vishay precision quarter bridge

- Biaxial Strain by a Vishay precision half bridge

- Shear Strain by a Vishay precision full bridge
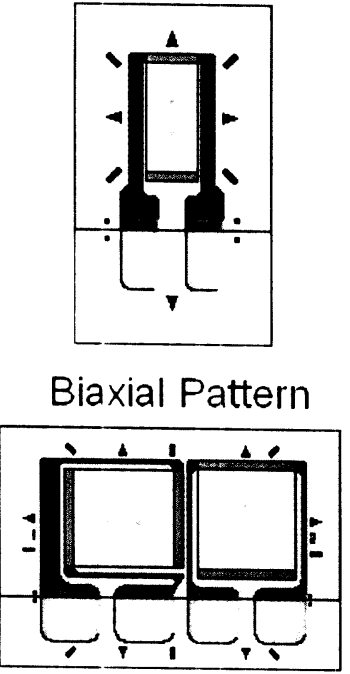

Shear Pattern

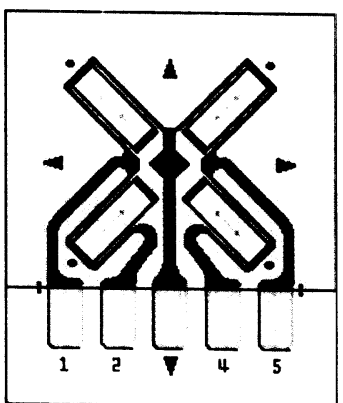




\section{Valve Health Monitoring System Instrumentation Types}

- Limit Switch Sensor

(for monitoring number of cycle)

- 6 magnetic reed switches

- 4 inputs with wake-up capabilities

- Signal Interface Sensor

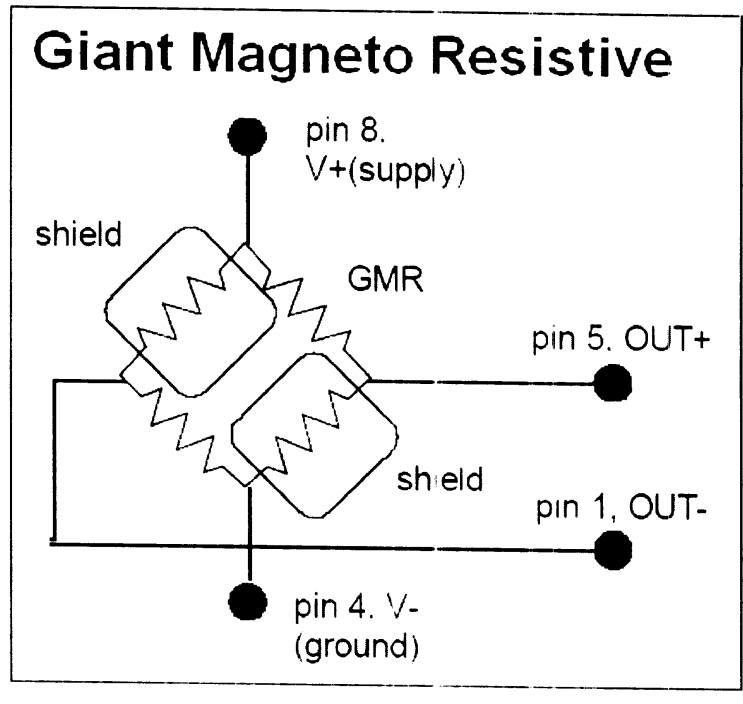

(for Linear Variable Displacement Transducer Transformer monitoring)

- 4 to 20 milliamp current loop signal

Giant Magneto Resistive (GMR) for

Unobtrusively monitors magnetic fields

- 0 to 10 volts Direct Current (DC) signal

Basic voltage follower circuit 


\section{Valve Health Monitoring System Conclusions}

The system is a feasible solution

- Can help maintain high-geared ball valves

- Can help maintain linearly actuated valves

- Flags degraded performance

- Generates operational performance history

- Able to be Integrated into a higher level health monitoring system

- Not a feasible detection system during a test 


\section{Valve Health Monitoring System Utilizing Smart Instrumentation}

\section{Questions}

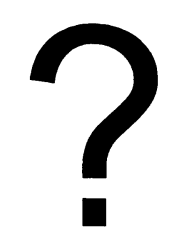




\section{Valve Health Monitoring System References}

- National Fire Protection Association National Electrical Code International Electrical Code Series, Quincy, Massachusetts USA 02169-7471.

- MG Chemicals Inc., www mcichem cals.com, 193rd Street, Surrey, BC, Canada V4N 4E7.

- Microchip Technology Inc. "PIC16F87XA Data Sheet 28/40/44-Pin Enhanced Flash Microcontrollers", ww microchip.Com, West Chandler Boulevard, Chandler, Arizona, USA 85224-6199.

- Linx Technologies Inc., www inxtechnologies.com, South East Ashley Place, Grants Pass, OR 975265.

- Measurement Specialties Inc., wwi meas-spec.com, Lucas Way, Hampton, VA 23666.

- Linear Technology Corporation, www vinear com, McCarthy Boulevard, Milpitas, CA 95035-7417.

- Analog Devices Corporation, wuw. analog.com, P. O. Box 9106,One Technology Way, Norwood, MA, 02062-9106.

- OMEGA Engineering Inc., wrw. omega.com, P. O. Box 4047, One Omega Drive, Starnford, Connecticut 06907-0047.

- Vishay Measurements Group Inc., P.O. Box 27777, Raleigh, NC 27611.

- NVE Corporation, www nve com, Valley View Road, Eden Prairie, MN 55344-3617.

- ARMA Design "Compact Flash Card Development Kit Manual”, Ferris Square, Suite E:, Sand Diego, CA 92121.

- ICS DataCom “I-7188E Hardware Manual”, Commerce Circle, Pleasanton, California 94588.

The use of trademarks or names of manufacturers in the report is for accurate reporting and does not constitute an official endorsement, either expressed or implied, of such products or manufactures by the National Aeronautics and Space Administration. 